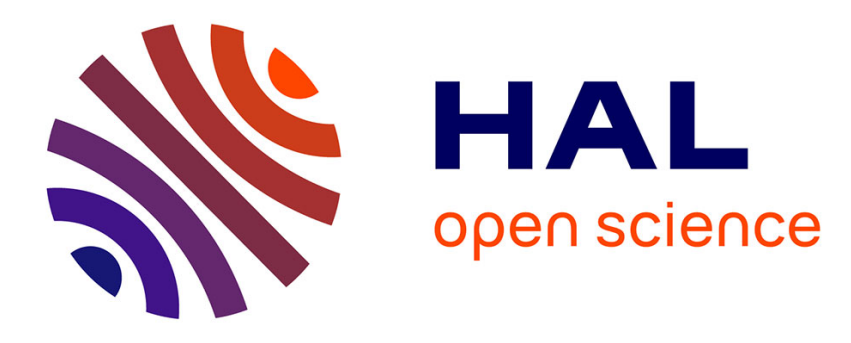

\title{
Retour historiographique sur l'iconographie du quotidien en Gaule
}

\author{
Jeanne-Marie Demarolle
}

\section{To cite this version:}

Jeanne-Marie Demarolle. Retour historiographique sur l'iconographie du quotidien en Gaule. XIVème colloque international d'art provincial romain: Iconographie du quotidien dans l'art provincial romain.

Modèles régionaux, Jun 2015, Dijon, France. hal-03550656

\section{HAL Id: hal-03550656 \\ https://hal.univ-lorraine.fr/hal-03550656}

Submitted on 1 Feb 2022

HAL is a multi-disciplinary open access archive for the deposit and dissemination of scientific research documents, whether they are published or not. The documents may come from teaching and research institutions in France or abroad, or from public or private research centers.
L'archive ouverte pluridisciplinaire HAL, est destinée au dépôt et à la diffusion de documents scientifiques de niveau recherche, publiés ou non, émanant des établissements d'enseignement et de recherche français ou étrangers, des laboratoires publics ou privés. 
DIJON : XIVème colloque sur l'art romain provincial

Jeanne-Marie DEMAROLLE

Professeur émérite à l'Université de Lorraine

Retour historiographique sur l'iconographie du quotidien en Gaule

Fidèle à une heureuse tradition d'alternance entre l'Orient et l'Occident de l'Empire le colloque sur l'art provincial romain s'éloigne de la Roumanie pour célébrer en France son XIVème anniversaire. Pour la deuxième fois ce colloque international biannuel se réunit dans une province gauloise: après la Narbonnaise en 2007, c'est cette année le tour de la Lyonnaise. Après Arles et Aix-en-Provence, prestigieuses cités antiques s'il en fut, le privilège d'accueillir tant de chercheurs venant d'horizons différents échoit à une "petite ville » lingonne, Divio.

C'est là une immense fierté pour tous ceux qui, depuis deux ans sont activement engagés dans la préparation de l'entreprise. L'Université de Bourgogne, le Musée archéologique, la ville de Dijon ont droit à notre sincère gratitude mais, derrière ces instances, saluons les chevilles ouvrières qui n'ont pas ménagé leurs efforts, tout particulièrement nos collègues Sabine Lefebvre Professeur à l'Université de Bourgogne et plusieurs enseignants chercheurs de l'UFR et de l'UMR ARTéHIS. N'oublions pas Simone Deyts dont la compétence en matière de sculpture gallo-romaine tant en pierre qu'en bois est inégalée.

Ajoutons aux universitaires, Christian Vernou et l'équipe du musée qui ont œuvré avec passion pour rassembler à Dijon quelques beaux exemplaires des reliefs dont nous allons débattre. Permettez-moi aussi de les remercier de m'avoir amicalement confié la responsabilité d'ouvrir cette session. J'attache beaucoup de prix à cet honneur mais, nul ne l'ignore, honos est onus. L'adage est d'autant plus vrai que, nul ne l'ignore non plus, je ne suis pas une spécialiste d'histoire de l'art.

Toutefois, les œuvres provinciales sur lesquelles repose la thématique du colloque 2015, si elles n'ont cessé depuis Drexel, Hahl, Koethe Schoppa, Hatt ou Gabelmann d'être interrogées pour leurs spécificités artistiques, et leur contribution à l'histoire de l'art gallo-romain l'ont été de plus en plus, depuis les années 1970, pour leur apport à l'histoire sociale, qu'il s'agisse d'activités économiques, de techniques, de comportements, de modes de communication.

D'autre part, longtemps condamnées à servir de simples illustrations en raison de leur valeur esthétique jugée médiocre au vu des canons de la "grande»sculpture, les figurations du quotidien sont largement entrées dans le champ des recherches sur la construction de l'image et le discours iconographique, laissant derrière elles les analyses historicistes de lecture immédiate au profit des apports de la sémiologie. C'est dans ces deux dernières perspectives que, de Metz où elles ont eu un grand succès auprès des Médiomatriques, je les ai moi-même abordées.

\section{Dijon et le XIV ${ }^{\mathrm{e}}$ colloque}

\subsection{Les scènes du quotidien et Dijon}

Avant même de retracer les grandes lignes de l'évolution historiographique, et plus particulièrement à propos de la présence du quotidien dans la sculpture sur pierre de la Gaule du Nord-est, je voudrais souligner combien le choix de Dijon et du visuel, même si le monument choisi a été retrouvé à une trentaine de kilomètres au nord de Dijon, est en parfaite 
adéquation avec le thème retenu, Iconographie du quotidien. Jusqu'à présent, en dépit de sa cohérence, celui-ci n'est apparu que de façon accessoire dans les treize colloques précédents. Mais, en parcourant les riches collections muséographiques et l'exposition Pax Romana, vous vérifierez une fois de plus combien dans l'espace qui s'étend des Bituriges aux Germanies, de l'Autunois au Soissonnais (Fig. 1), ce thème a rencontré une réelle faveur dans la sculpture régionale. Scènes de repas, de métier, de toilette, de voiturage mais aussi couples et familles dans leur gestuelle, leur tenue et leur parure, outils, instruments de musique autant d'éléments qui permettent de brosser des tableaux concrets et expressifs. Ils doivent beaucoup dans toutes les cités antiques de cette grande région, en Bourgogne mais aussi en Champagne et en Lorraine, à l'abondance sur les plateaux jurassiques d'une pierre de grande qualité, d'extraction facile.

Pour ce XIVème anniversaire, Fortuna elle-même semble avoir favorisé Dijon. Vous vous en souvenez, en ouvrant à Arles le Xème colloque Manfred Hainzmann appelait de ses vœux une collaboration plus étroite entre épigraphistes et historiens d'art. Depuis, la publication par l'archéologue S. Venault et par Y. Le Bohec (pour les inscriptions) et S. Deyts (pour la sculpture) des découvertes faites à Autun à la nécropole de Pont-l'Evêque répond pleinement à cette attente. On peut féliciter les auteurs de la célérité avec laquelle ils ont mené à bien l'étude épigraphique et iconographique des monuments que l'excursion prévue au programme vous permettra d'apprécier. Pour rester dans le domaine de l'épigraphie mais sans quitter Dijon, on sait combien les marmorarii, lapidarii, sculptores/ scalptores et autres artisans de la pierre ont été des plus discrets dans le monde romain.

Or, Divio a la chance de posséder l'inscription votive adressée à Iuppiter Optimus Maximus et à Fortuna redux pour la préservation «pro salute » de leur patron par ses clients lapidarii, et à Autun en 2004 a été dégagée l'épitaphe sur un autel funéraire de l'operarius Sacilus lapidarius .

\subsection{Le monument du marchand de vin, représentatif du contexte des recherches}

Le monument dit «du marchand de vin », si souvent reproduit mais si souvent aussi de manière partielle, permet de revenir sur un certain nombre de réserves méthodologiques qui semblent nécessaires pour un usage véritablement scientifique du quotidien dans l'art provincial. Particulièrement étudié par $S$. Deyts, un des plus célèbres de la belle collection du musée de Dijon, il est tout à fait emblématique à plus d'un titre des scènes du quotidien en Gaule romaine ou, plus exactement, des scènes du quotidien telles qu'elles sont figurées dans l'art gallo-romain et telles qu'elles apparaissent dans le discours scientifique. Les hasards des réemplois et des découvertes ont fait parvenir jusqu'à nous une «scène de rue » (Fig. 2 et 3).

De prime abord, il est représentatif de l'état des documents livrés à l'exégèse, jamais intacts rarement complets, ayant souvent perdu leur inscription et la polychromie susceptible tout à la fois de «masquer» des défauts mais aussi, ce qui n'est pas négligeable en termes de réception des images, d'attirer le regard sur tel ou tel détail. C'est là un fragment d'un monument funéraire, type de lapidaire qui piliers, autels, cippes, stèles confondus est le support privilégié, sinon des représentations du quotidien (les mosaïques, les enduits peints ont eu également recours à cette thématique) tout au moins des analyses les concernant.

Or, appartenir à la catégorie funéraire introduit nécessairement des interrogations sur le symbolisme, sur les rapports entre les morts et les vivants, interrogations qui, au-delà de la lecture immédiate, font-elles aussi partie du quotidien. Son iconographie place le monument $\mathrm{du}$ «marchand de vin » dans la série bien fournie d'images de la vie «courante », et de sur crôt dans la catégorie la plus riche, celle des scènes d'artisanat et de commerce. 
Retrouvé à une trentaine de kilomètres de Dijon, à Til-Châtel, le monument du marchand de vin provient d'un milieu urbain, d'une agglomération secondaire Il est ainsi tout à fait représentatif de l'état de la question, puisque la majorité des monuments sculptés de ce type ont été exhumés dans des centres urbains, capitales de province (Reims) ou de (cité Langres, Metz, Trèves, Besançon) mais aussi d'agglomérations secondaires (Dijon certes, mais aussi Arlon, Auxerre, Saulieu, Saverne, La Bure, Soulosse, Entrains, Le Hérapel...). Aujourd'hui, le corpus mérite certes d'être jugé «urbain » mais c'est là d'abord un effet de source. La colonne d'Igel, seul monument de ce type ainsi décoré encore in situ et les récentes découvertes de Durbach dans l'Eifel suffisent à prouver que les riches propriétaires fonciers, pour leurs imposants mausolées, ont puisé non seulement dans la sculpture mythologique érudite mais aussi dans les réalités plus ordinaires. Pas plus ici qu'en Italie (Zimmer). l'iconographie dite du quotidien ne saurait être considérée comme l'apanage d'un «art plébeien » et des seules catégories moyennes de la population. Au même titre que les scènes de toilette étudiées par J. Guerrier-Delclos), celle-ci, contrairement à la règle générale, ne semble pas inspirée de modèles venant de Rome ou d'Italie.

Mais le monument ne laisse pas voir, qu'il provient d'une nécropole de la station de Tilena, mentionnée sur la carte de Peutinger, alors que la grande majorité du corpus a été réemployée pour édifier des remparts. Issu de découvertes anciennes, dépourvu de tout contexte stratigraphique et d'inscription ce monument témoigne bien des difficultés ordinaires de datation d'une grande partie de l'iconographie du «quotidien », presque toujours faite de membra disiecta. D'autres scènes et lesquelles complétaient-elles celle-ci, obligeant à d'autres interprétations, à d'autres conclusions, à d'autres restitutions ?

Bref, d'évidence le monument du marchand de vin et pas davantage le petit millier de représentations du «quotidien » et plus encore les études menées ne montrent qu'une partie du «quotidien ». En effet, deux pans importants de la vie des Gallo-romains tiennent peu de place, la vie religieuse et le loisir. Dans ce monde d'images, le negotium l'emporte largement sur l'otium. Par ailleurs, dans la mesure où l'essentiel des représentations se situe du milieu du IIe siècle au milieu du IIIè siècle, c'est en extrapolant que nous parlons des scènes $d u$ quotidien en Gaule romaine. Autrement dit, les chercheurs ne disposent, en fait, que d'une séquence chronologique et d'une vision partielle. Pourtant, il a longtemps été admis que ces scènes correspondaient à autant d'instantanés pris sur le vif à Divio, à Augustodunum, à Agedincum, à Divodurum... Or, l'historiographie d'un demi-siècle de recherches incite à nuancer le propos.

\section{Un demi-siècle de recherches sur l'iconographie du quotidien}

Même si dans La tombe gallo-romaine J.-J.Hatt s'était intéressé à ce type de représentations, si F. Braemer avait analysé de près le corpus de Bordeaux et si le banquet funèbre avait suscité d'importantes études outre-Rhin, c'est à partir des années 1970 que la thématique du quotidien a fait naître un nombre considérable d'études fructueuses. Toutefois, dans le cadre des colloques sur l'art provincial elle n'a jamais été abordée pour elle-même.

Il ne saurait être question ici de traiter de toute la Gaule et nous nous limitons aux recherches, et elles sont fort nombreuses, portant sur le Nord- est. Mais, même dans cet espace, aucune synthèse sur l'ensemble des reliefs conservés n'a encore abouti. Les travaux majeurs portent, en effet, soit sur le secteur rhénan et un type de scène (repas funèbre, repas de famille, Noelke, Colling) soit sur les scènes d'artisanat et de commerce (Reddé, Pannoux, Béal, Demarolle,Langner), soit sur tous les types de scènes mais dans un espace restreint : ( Baltzer, Freigang). 


\section{1 : L'apport des recherches en histoire de l'art}

La priorité a longtemps été donnée à la grande sculpture architecturale et en ronde bosse qui offre des critères stylistiques de datation plus apparents. Quant aux classifications établies depuis M. Baltzer elles ont avant tout pris en compte la typologie des monuments (stèles, piliers, autels), celle de la représentation des défunts (en pied, en buste), celle des décors architecturaux, et les catégories de sujets (vie privée, vie professionnelle) dans une double perspective, affiner les datations (Baltzer, Numrich), établir la «généalogie » de la figuration, mesurer la part respective de l'apport « romain » et du fonds « indigéne ».

Plus récemment, le travail des chercheurs a porté essentiellement sur l'identification et la datation d'ateliers régionaux, en Bourgogne du sud, chez les Séquanes, chez les Bituriges et chez les Leuques. En scrutant les rapports entre la sculpture architecturale et la sculpture funéraire (Hatt, Deyts, Walter), en s'attachant de plus près à des détails techniques (Deyts, Coulon-Deyts, Moitrieux, Castorio), une distribution géographique des ateliers et des transferts entre ateliers des capitales (Trèves, Autun) et ateliers des vici (Nuits Saint-Georges, Arlon,) ont pu être mis en évidence ici (style de la Cöte) et dans les Germanies (Noelke). De jeunes chercheurs de l'Université de Bourgogne, qui sont intervenus au colloque prennent la relève, sur les ateliers de sculpture des Eduens et des Lingons et sur les monuments funéraires de ces deux cités.

L'ensemble des travaux souligne l'inventivité des artisans aussi bien dans la typologie des monuments (cippe demi-cylindrique propre à une partie de la Trévirie) que dans la gestuelle. Il met aussi en valeur l'importance des variations régionales (contrairement aux Trévires et aux Médiomatriques, les Eduens, les Lingons et les Séquanes ont peu employé les éléments achitectoniques) et celle des contraintes «techniques ». Représenter les personnages en buste (Fig. $4^{\circ}$ ) plutôt qu'en pied dépend peut-être plus de la difficulté de tailler une pierre dure que du choix délibéré d'un modèle italien.

Pour l'instant, toutes les régions ne bénéficient pas d'inventaires exhaustifs, ce qui limite l'avancée des investigations sur la mobilité des artisans et la diffusion de cartons à l'échelle de grands ensembles, sachant toutefois que nombre d'artisans dont on retrouve des monuments en cours d'exécution dans de modestes sites campagnards ne sont intervenus que localement.

\section{2. : L'apport des recherches en histoire socio-culturelle}

Les historiens de la société gallo-romaine, et ce dès Camille Jullian, ont vu quant à eux dans les scènes du quotidien une source magistrale documentant les croyances funéraires, le monde du travail, la célébration sociale.

C'est essentiellement l'iconographie de la face antérieure, où les défunts apparaissent dans des poses (en pied, en buste, à table), des tenues, des gestuelles et avec des attributs stéréotypés (poculum, mappa, flacon, tablettes...) qui a suscité les interrogations et alimenté les débats à propos par exemple du gobelet que tiennent les défunts) en glissant du registre symbolique de la mort et de la vie future à celui de l'identité culturelle.

Mais c'est le décor de l'ensemble du monument qui a été pris en compte pour étudier les deux autres domaines (Reddé, Pannoux, Freigang Béal,, Noelke, Demarolle, Colling) en privilégiant deux sujets, les scènes de repas et les scènes de métier. Ces travaux ont eu pour contexte de recherche les hypothèses sur la culture «plébeienne" de Renato BianchiBandinelli et de Paul Veyne, la thèse de G. Zimmer qui les réfute, le développement d'une histoire sociale de l'art et les propositions de P. Zanker sur la nature sociologique des images 
romaines, avant de tirer parti des pistes ouvertes en matière de sémiologie des images par $\mathrm{R}$. Barthes et T. Hölscher.

Ces travaux ont mis en évidence que ces représentations sont autant de "mises en scène » hybrides. Elles associent à des éléments précis de réalisme (validés par l'archéologie, voire par l'archéologie expérimentale pour le vallus) indispensables pour assurer la lisibilité des messages à divers codes à l'œuvre dans tout l'Empire (enfant entre ses parents, tibicen tenant son instrument au lieu d'en jouer ...). Les éléments réalistes témoignent de l'inventivité des artisans qui n'ont pas copié servilement les modèles venus d'Italie et de l'attachement au fonds autochtone.

Mais il ne faut pas s'attendre à un panorama complet du monde professionnel puisque quelques secteurs (travail, du métal, du bois, de la pierre, du textile) ont été privilégiés, que les scènes de commerce l'emportent sur celles de production, que le monde agricole est pratiquement absent. Le niveau économique des activités (de modestes artisans, des « entrepreneurs » (Fig. 5 et 6) et le niveau social des commanditaires, uniformément drapés dans des vêtements « indigènes », soucieux d'une respectable autocélébration, restent objet de débats (Béal, Freigang)

Ces approches, loin d'être exclusives, sont complémentaires. Les scènes de voiturage empruntent au quotidien la caisse tressée d'osier et aux conventions esthétiques le pas de parade des chevaux (Raepsaet) mais « récupèrent » peut-être aussi dans les pays celtes (Gaule, Pannonie) à l'époque romaine une partie de la symbolique du char de combat de l'aristocratie celte (Freigang).

\section{Et maintenant ? En guise de conclusion...}

Tous les deux ans les colloques sont l'occasion, non seulement d'approfondir le thème retenu mais aussi de revenir sur des questions d'importance fondamentale pour les progrès des recherches, questions liées à de récurrentes difficultés.

\subsection{Relectures}

Reprendre sans relàche la lecture de l'iconographie du quotidien dans un souci constant d'approfondir- voire de renouveler les identifications et les interprétations est une nécessité. Deux exemples récents peuvent étayer mon propos. Les enfants de la scène dite « repas des parents et des enfants (Fig. 7) sur un cippe d'Arlon viennent d'être reconnus par C Vendriès comme de jeunes esclaves, dont un tibicen. On passe d'un repas célébrant la famille au sens d'aujourd'hui à une scène mettant en exergue la familia, l'aisance, la culture « romaine ». En revenant sur la stèle tout aussi célèbre de Severina, la nutrix de Cologne, U. Rothe vient de démontrer que le monument a été érigé pour Severina et non par elle.

\section{2. Qu'attendre de l'épigraphie?}

Le monument de Cologne précise le métier de Severina, tout comme celui de Marcellus (Fig.8) à Metz. Dans ces deux exemples, l'inscription complète l'image : la représentation de Marcellus, arborant fièrement chaîne et cadenas, ne permet pas de trancher : est-il fabricant, est-il fabricant/ commerçant ? La dédicace fait voir en lui un marchand en gros puisqu'il se présente comme negotiator artis clostrariae. Malheureusement, la majorité des inscriptions est très laconique et ne précise pas souvent le métier exercé. Et lorsqu'elle le fait la polysémie des mots (ex. vestiarius) ne facilite pas l'interprétation. En matière de datation, en dépit de tous les progrès, le chercheur doit se contenter de larges fourchettes : $1^{\text {ère }}$ moitié du IIè siècle pour les stèles d'Autun (Pont-l'Evêque), d'environ 150 à environ 240 pour la majorité des reliefs 


\subsection{De nécessaires élargissements}

Des élargissements semblent souhaitables, pour mieux appréhender le quotidien de la Gaule romaine, puisque les colloques portent sur l'art provincial dans ses diverses expressions.

Au-delà de la sculpture funéraire (cippes, stèles, piliers, sarcophages), la sculpture religieuse mérite d'être prise en considération. En dépit d'une nouvelle découverte l'identification de la scène (Fig. 9) retrouvée à Grand au XIX è siècle (divinité ?, fabrication de savon, de remèdes de fromage ?) reste incertaine.

Et d'autres types de supports (sculpture en bois, enduits peints, mosaïques, céramique sigillée et médaillons d'applique), permettent d'enrichir les données et les confrontations. Enfin, d'autres aspects de l'iconographie du quotidien doivent être abordés et ce sera justement un des acquis majeurs de cette rencontre puisque des communications sur le sport, l'ars medica, le quotidien du soldat, la vie intellectuelle sont annoncées. Ces élargissements ne manqueront pas de mettre en exergue, à l'instar des sujets déjà bien étudiés, les valeurs d'adoption et d'adaptation qui font la richesse de la « romanisation ».

\subsection{De nouveaux instruments de recherche}

Pour si diversifiées qu'elles soient les scènes du quotidien que donnent à voir les reliefs du Nord-est de la Gaule ne peuvent livrer tout leur potentiel scientifique qu'en les replaçant dans le cadre de l'Empire pour constituer des séries et multiplier les comparaisons pertinentes. Nous savons ce que nous devons aux remarquables entreprises du CSIR et du Nouvel Espérandieu. En publiant voici cinq ans le volume consacré à Toul et à la cité des Leuques, G. Moitrieux et J.-N. Castorio ont quintuplé le nombre de notices rassemblées par le commandant Espérandieu et le progrès, bien entendu, n'est pas que quantitatif. Un tel travail fait cruellement ressentir l'absence de volumes semblables pour les autres cités du Nord-est. Mais ayons foi dans l'avenir : le Nouvel Espérandieu, dirigé par H. Lavagne, doit compter vingt volumes, Internet propose deux instruments de travail : la base VBI ERAT LVPA et 1 «ancien Espérandieu » en ligne ; par ailleurs, les collections de nombreux musées sont en cours de numérisation. Mais l'accroissement des données ne portera ses fruits que si les colloques sur l'art provincial favorisent les décloisonnements entre histoire de l'art et histoire, entre épigraphie et iconographie et si l'audience des bibliographies dépasse les frontières nationales. Il est grand temps maintenant de donner la parole aux spécialistes.

Eléments de bibliographie

Baltzer M., „Die Alltagsdarstellungen der treverischen Grabdenkmäler. Untersuchungen zu Chronologie, Typologie und Komposition“", TZ, 46, 1983, p.7-151.

Barthes R., «Eléments de sémiologie », Communications, 4, 1964, p. 91-165.

Béal J.-Cl., «Pistor et materiarius : à propos d'une stèle funéraire de Metz antique », RAE, 47, 1996, p. 79-95.

Béal J.-Cl. , «Remarques sur l'imagerie du pilier funéraire d'Igel » dans Imago Antiquitatis. Religions et iconographie du monde romain. Mélanges offerts à Robert Turcan, Paris, 1999, p. 95-104.

Béal J.-Cl., «La dignité des artisans : les images d'artisans sur les monuments funéraires de Gaule romaine », $D A H, 26,2000$, p. 149-182.

Bohne A., Sportszene Untersuchungen zur Ikonographie römischer Athleten Darstellungen, Hildesheim, 2011.

Brilliant R., « Some reflections on the «new Roman art history » », JRA, 11, 1998 p. 557-564 Castorio J.-N., «Remarques sur la typologie des monuments funéraires de la cite des Leuques : emprunts et originalités », dans Noelke P., et alii ( éd.), Romanisation and 
Resistenz in Plastik, Architektur und Inschriften der Provinzen des Imperium Romanum : neue Funde und Forschungen, Mainz, 2003, p. 233-243

Castorio J.-N., « Le fonctionnement des ateliers de sculpture dans les agglomérations secondaires gallo_romaines », dans Delfosse P. (éd.), Hommages à Carl Deroux, IV, Bruxelles, 2003, p. 35-49

Castorio J.-N., «La sculpture d'époque romaine dans le sud de la Gaule mosellane ateliers, styles, chronologie » dans Gaggadis-Robin, Vassiliki et alii (éd.), Les ateliers de sculpture régionaux : technique, styles et iconographie, Arles, Aix- en- Provence, 2009, p. 565-573

Castorio J. N. et Maligorne Y., «les monuments funéraires dans le sud de la Gaule mosellane » dans Reddé M. et alii (éd.), Aspects de la romanisation dans l'est de la Gaule, vol. 2, Glux-en Glenne Bibracte, 2011, p. 791-802

Chauch D., Etude iconographique des stèles funéraires gallo-romaines de Sens à Dijon: éléments communs, convergences stylistiques, ateliers Mémoire de maîtrise, Université de Bourgogne, Dijon, 1998

Colling D., «Les scènes de banquet funéraire ou Totenmahlreliefs originaires d'Arlon », Bulletin trimestriel de l'Institut Archéologique du Luxembourg, 87, 2011, p. 155-176

Coulon G., et Deyts S., Les stèles funéraires gallo-romaines de Saint-Ambroix (Cher). Un atelier de sculpture dans la cité des Bituriges, Lancosme multimedia et les musées de Châteauroux, 2012

Demarolle J.-M., «Igel et Metz : à propos d'une nouvelle scène d'examen du tissu », Annales de l'Est, 2001 p. 63-81

Demarolle J.-M., «Un corpus en question, l'iconographie lapidaire des métiers en Gaule Belgique », dans Polfer M. (éd.), L'artisanat romain, évolutions, continuités et ruptures (Italie et provinces occidentales), Montagnac 2001, (Monographies Instrumentum 20) p. 3142

Demarolle J.-M., «Epigraphie et iconographie : l'expression de la promotion sociale sur les monuments funéraires de la Gaule Belgique », dans Bedon R. et Polfer M. (éd.), Etre Romain, Hommages in memoriam Charles Marie Ternes, Remshalden, 2007, p. 349-367

Deyts S., «Recherches sur les ateliers de sculpture gallo-romaine dans la région de Dijon », $R A E, 22,1971$, p. 353-391

Deyts S., Sculptures gallo-romaines mythologiques et religieuses, Dijon, Musée archéologique, Inventaire des collections publiques françaises n²0, Paris, 1976

Deyts S., Un peuple de pélerins : offrandes de pierre et de bronze des Sources de la Seine $\left(13^{\text {ème }}\right.$ suppl. à la $\left.R A E\right)$, Dijon, 1994

Deyts S., Les bois sculptés des sources de la Seine (42 suppl. à Gallia), Paris, 1983

Deyts S., «Ateliers de sculpture d'Autun, Langres et Dijon » dans Chardron-Picault P. (dir.), Aspects de l'artisanat en milieu urbain: Gaule et Occident romain ( $28^{\mathrm{e}}$ suppl. à la $\left.R A E\right)$, 2010, p. 281-287

Deyts S. «A propos des marchands de vin du Musée archéologique de Dijon », Bulletin des Musées de Dijon $\mathrm{n}^{\circ} 13$, p. 19-23

Deyts S. "Ateliers de sculpture antique du pays éduen (d'Autun à Saulieu et à Nuits-SaintGeorges), Mémoires de la Commission des Antiquités du département de la Côte-d'Or, 41, 2005-2011 (2014), p. 30-45

Deyts S., Barçon, J.-Cl., «Un type de monument funéraire original : le marchand de vin du musée archéologique de Dijon» dans Hommages à Lucien Lerat, Besançon (ALUB 294) Paris, 1984, p. 241-252

Faust W., Die Grabstelen des 2. Und 3. Jahrhunderts im Rheingebiet (BJb Beiheft 52), Köln, 1998 
Freigang Y.1997, «Die Grabmäler der gallo-römischen Kultur im Moselland. Studien zur Selbstdarstellung einer Gesellschaft", JRGZM, 44, p. 277-440

Gabelmann H., „Die Typen der römischen Grabstelen am Rhein“, BJb, 172, 1972, p. 65-140

Hahl L; Zur Stylentwicklung der provinzial römischen Plastik in Germanien und Gallien, Darmstadt, 1937

Hatt J.-J., «Esquisse d'une histoire de la sculpture régionale de Gaule romaine principalement dans le Nord-est de la Gaule », REA, 59, 1957, p. 76-107

Hatt J.-J., Sculptures antiques régionales, Musée archéologique de Strasbourg, Paris, 1964

Hatt J.-J., La tombe gallo-romaine, Paris, 1986 (2è éd.)

Hölscher T., Bildsprache als semantisches System, 1978

Hope V.M., Constructing Identity. The Roman Funerary Monuments of Aquileia, Mainz and Nîmes (BAR Inter.Ser. 960) Oxford, 2001

Huet V., «Les images du sacrifice en Gaule romaine » dans Lepetz S., van Andringa W., (éd.), Archéologie du sacrifice animal en Gaule romaine. Rituels et pratiques alimentaires, Paris, 2008, p. 43-74

Jockey Ph., "Les artisans de la pierre dans le monde gallo-romain et leur éventuelle exploitation par l'historien", Topoi, 8,1998, p. 625-652

Kazek K;Gladiateurs et chasseurs en Gaule. Au temps de l'arène triomphante Ier-IIè siècle apr. J.-C., Presses Universitaires de Rennes, 2012

Koethe H., «La sculpture romaine au pays des Trévires », $R A, 1937$, p. 199-239

Kristensen T. M. and Poulsen B. (éd.), Ateliers and artisans in Roman art and archaeology (JRA Supplementary series 92), 2012

Langner M.," Szenen aus Handwerk und Handel auf gallo-Römischen Grabmälern“, JDAI, 116,2001 , p. 301-356

Laubry N., „Aspects de la romanisation en Gaule et en Germanie: les inscriptions et les monuments funéraires sous le Haut Empire“, Pallas, 80, 2009, p. 281-305“

Lissarague F. et Rosso E., «Entretien avec Tonio Hölscher », Perspective, 2014, p. 167-180

Marfaing K, Traits de particularisme et éléments communs dans la sculpture funéraire galloromaine en Bourgogne et Franche-Comté : La representation des défunts sur les stèles, Mémoire de DEA dirigé par H. Walter, Besançon, 1997, 2 vol.

Misciatelli E. 1981, «Les monuments funéraires de Reims gallo-romain, catalogue des monuments figurés du Musée St-Rémi et d'autres collections », Bulletin de la Société archéologique champenoise $\mathrm{n}^{\circ} 4$

Molin M., "Quelques considérations sur le chariot des vendanges de Langres (HauteMarne) », Gallia, 42, 1984, p. 97-114

Moitrieux G. et Castorio (J.-N.), Nouvel Espérandieu III, Toul et la cité des Leuques, Paris, 2010

Nerzic C., La sculpture en Gaule romaine, Paris, 1989

Nümrich B., Die Architektur der römischen Grabdenkmäler (TZ Beiheft 22), Trier, 1997

Noelke P. Zur Chronologie der Grabreliefs mit Mahldarstellung im römischen Germanien“dans Walter 2000, p. 59-70

Noelke P., „Zu den Grabreliefs mit Darstellung des convivium coniugale im römischen Germanien und im benachbarten Gallien, BJb 205, 2005, p. 155-241

Noelke P., «Bildhauerwerkstätten im römischen Germanien „“ BJb 206, 2006, p. 87-144

Ormasti M. R., Iconographie des stèles funéraires et des blocs mis au jour à l'îlot SaintJacques (Metz), Ier-IIIè siècles, Mémoire de maîtrise, Université de Metz, 1992

Pannoux S., «La représentation du travail : récit et image sur les monuments funéraires des Médiomatriques », DAH, 11, 1985, p. 293-328 
Raepsaet G. «Les attelages d'Igel et l'expérimentation des harnais gallo-romains », Annales de l'Est, 2001, p. 27-41

Reddé M., «Les scènes de métier dans la sculpture funéraire gallo-romaine », Gallia, 36, 1978, p. 43-63

Rothe U., Dress and Cultural Identity in the Rhine-Moselle Region of the Roman Empire, ( $B A R$ Internat. Ser. 2038), Oxford, 2009

Rothe U., « Der Grabstein der Severina Nutrix aus Köln. Eine neue Deutung », Germania, 89, 2011, p. 191-214

Schwinden L; 2001, «Les scènes dites de «paiement du fermage ou « scènes de comptoir » de la colonne d'Igel et des monuments de Trèves », Annales de l'Est, 2001, p. 55-62

Stewart P., «Totenmahlreliefs in the northern provinces : a case study in imperial sculpture », $J R A, 22,2009$, p. 252-274.

Tarpin M., "Modèles italiens et ateliers indigènes. Un exemple lyonnais », dans Solin H., Salomies O., Liertz U.M. '(éd.), Acta colloquii epigraphici Latini Helsingae habiti 3-6 sept. (Commentationes Humanarum litterarum 104), Helsinki, p. 347-372

Vendriès C., «Ventre affamé n'a pas d'oreilles ? La représentation d'un esclave tibicen dans une scène de banquet sculptée sur un cippe d'Arlon », L'Antiquité classique, 70, 2001, p. 123136

Vendriès C., «Une stèle de musicien dans la nécropole de Pont-l'Eveque à Autun. Le tibicen Brunnius », Latomus, 72, 2013, p. 1022-1031

Venault S., Deyts S., Le Bohec Y., Labaune Y., «Les stèles funéraires de la nécropole de Pont-l'Evêque, contexte de découverte (YL, SV) et étude du corpus (SD, YLB), Bulletin archéologique du CTHS, 35 , 2009, p. 129-201.

Walter H., La sculpture funéraire gallo-romaine en Franche-Comté, ALUB 156, BesançonParis, 1974 et « Supplément à la sculpture funéraire », RAE, 30, 1979

Walter H., (éd.), La sculpture d'époque romaine dans le nord, dans l'est des Gaules et dans les régions avoisinantes : acquis et problématiques actuelles, Actes du colloque de Besançon 1998, ALUB ,694, Paris, 2000

Wild J. P., "Textiles et activités relatives au textile sur le monument d'Igel”, Annales de l'Est, 2001, p. 83-92

Willer S., Römische Grabbauten des 2.und 3.Jahrhunderts nach Christus im Rheingebiet.(Beihefte der Bonner Jahrbücher 56), 2005.

Young A., „Representations of Cloth vendors and the Cloth Trade on Funerary Reliefs in Roman gaul and Italy“, dans Cardon D. et Feugère M.éd)., Archéologie des textiles des origines au Vè siècle. Actes du colloque de Lattes, oct. 1999, (Monographies Instrumentum 4), Montagnac, 2000, p. 215-235

Zenarolla I „Il relievo gladiatorio conservato a Triesto. Una rilettura“, Atti Mem Istria, 101, 2001, p. 129-142

Zimmer G., Römische Berufsdarstellungen, Berlin, 1982

\section{Illustrations}

Fig. 1 : Localisation de représentations lapidaires en Gaule du Nord-Est.

Fig. 2 et 3 :Le monument (mutilé) du marchand de vin de Dijon et propositin de restitution de 1'ensemble par S.Deyts.

Fig. 4 : Défunts en buste (nécropole d'Autun, Pont-l'Evêque).

Fig. 5 et 6 : Faces latérales du monument funéraire messin d'un entrepreneur, materiarius et pistor (@Musée de la Cour d'Or Metz Métropole/L. Kieffer) 
Fig. 7 : Face principale du monument funéraire de Marcellus (CMusée de la Cour d'Or Metz Métropole/L. Kieffer)

Fig. 8 : Cippe d'Arlon (CMusée de la Cour d'Or Metz Métropole/L. Kieffer)

Fig. 9 : Scène religieuse ( ?) retrouvée à Grand au XIX è siècle (Musée d'Epinal.@ MDAAC Epinal. Photo J. Laurençon, CGV) 